\title{
Minimal Weighted Local Variance as Edge Detector for Active Contour Models
}

\author{
W.K. Law and Albert C.S. Chung \\ Lo Kwee-Seong Medical Image Analysis Laboratory, \\ Department of Computer Science, \\ The Hong Kong University of Science and Technology, \\ Clear Water Bay, Kowloon, Hong Kong \\ \{maxlawwk, achung\}@cs.ust.hk
}

\begin{abstract}
Performing segmentation of narrow, elongated structures with low contrast boundaries is a challenging problem. Boundaries of these structures are difficult to be located when noise exists or intensity of objects and background is varying. Using the active contour methods, this paper proposes a new vector field for detection of such structures. In this paper, unlike other work, object boundaries are not defined by intensity gradient but statistics obtained from a set of filters applied on an image. The direction and magnitude of edges are estimated such that the minimal weighted local variance condition is satisfied. This can effectively prevent contour leakage and discontinuity by linking disconnected boundaries with coherent orientation. It is experimentally shown that our method is robust to intensity variation in the image, and very suitable to deal with images with narrow structures and blurry edges, such as blood vessels.
\end{abstract}

\section{Introduction}

Active contour models are widely used in solving medical image segmentation problems. For instance, blood vessel segmentation is one of the applications in medical image segmentation. To separate vascular structures from the image background, researchers consider utilizing image gradient as a criterion to label blood vessel boundaries. In the Gradient Vector Flow (GVF) method [1, a moving parametric contour is driven by the minimization of energy $\mathcal{E}$,

$$
\mathcal{E}(\mathcal{C})=\iint \mu\left(u_{x}^{2}+u_{y}^{2}+v_{x}^{2}+v_{y}^{2}\right)+|\nabla f|^{2}|\mathbf{v}-\nabla f|^{2} d x d y,
$$

where $f=|\nabla I(x, y)|^{2}$ represents the edge map of an image $I, \mathbf{v}(x, y)=$ $(u(x, y), v(x, y))^{T}$ denotes the flow vector, which is obtained using two diffusion based partial differential equations in the whole image domain, $\mu \nabla^{2} u-$ $\left(u-f_{x}\right)\left(f_{x}^{2}+f_{y}^{2}\right)=0$ and $\mu \nabla^{2} v-\left(v-f_{y}\right)\left(f_{x}^{2}+f_{y}^{2}\right)=0$. As such, the diffusion process creates a competition of forces exerting from the image gradient at different locations. The GVF method outperforms the classical Snakes [2] because 
the above diffusion processes enable GVF to have long range interaction between boundaries and moving contours.

Apart from the parametric contours, Malladi et at. 3] have proposed to use the level set framework [4 for modeling of moving curves. The level set formulation can handle merging or splitting of contours naturally. One of the main ideas in [3] is that there is an advection term, which keeps the front of the level set function expanding (or contracting) with the speed controlled by a function, namely edge detector, $g(\nabla I(x, y))=\frac{1}{1+\left|\nabla G_{\sigma} * I(x, y)\right|^{p}}, p \geq 1$. This formulation keeps the contours exploring the image and eventually halted on the object boundaries, where the edge detector gives small value.

However, these methods are not suitable for elongated or low contrast objects such as blood vessels in the brain. For example, for the GVF method, it favors the conceptual edges and usually discards the narrow regions rather than including them in the segmentation results. Also, the edge detector relies on high image gradient magnitude to halt the moving contours, and can fail to detect low contrast boundaries.

To deal with this problem, Vasilevskiy et al. proposed the use of flux maximizing geometric flows for image segmentation [5]. Different from the above methods, object boundaries are detected by incorporating image gradient direction and magnitude. The contour motion is governed by

$$
\mathcal{C}_{t}=\nabla(\mathcal{V}(x, y)) \mathcal{N}
$$

where $\mathcal{V}(x, y)$ is the gradient vector of an image and $\mathcal{N}$ is the normal direction on the curve $\mathcal{C}$. Contour evolution direction is guided by the direction perpendicular to the image gradient. It does not fail in the situation where gradient magnitude is small or object structures are elongated and thin.

Along the same line, Xiang et al. introduced an elastic model [6] for segmentation of thin concave and convex structures. This method also integrates the information of both the magnitude and direction of image gradient in the similar fashion. In 6], the image gradient magnitude is extended to whole image domain rather than locally defined, as in [5]. The dynamics of an active contour is defined by minimizing the energy,

$$
\mathcal{E}(\mathcal{C})=\frac{1}{2} \int \mathbf{w} \cdot \mathbf{w} d x d y d z
$$

subject to the constraint,

$$
\nabla \times \mathbf{w}=\delta_{\mathcal{C}} \mathbf{t}
$$

where $\mathbf{w}$ is a three dimensional vector field, $\delta_{\mathcal{C}}$ is Dirac delta-function which is zero everywhere except on the curve $\mathcal{C}$. $\delta_{\mathcal{C}} \mathbf{t}$ is approximated by $\delta(z)$. $\left(\frac{\partial\left(G_{\sigma} * I\right)}{\partial y},-\frac{\partial\left(G_{\sigma} * I\right)}{\partial x}, 0\right)^{T}$ such that $\mathcal{C}$ can be attracted towards the object boundaries by minimizing the energy above.

On the other hand, without considering image gradient, Chan and Vese suggested to perform image segmentation by solving the minimal partition problem in [7. The segmentation result is the minimizer of an energy functional, 
$F\left(c_{1}, c_{2}, \mathcal{C}\right)=\mu \mathcal{C}_{\text {Length }}+\nu \mathcal{C}_{\text {Area }}+\lambda_{1} \int_{\mathcal{C}_{\text {in }}}\left|I-c_{1}\right|^{2} d x d y+\lambda_{2} \int_{\mathcal{C}_{\text {out }}}\left|I-c_{2}\right|^{2} d x d y$,

where $\mu \geq 0, \nu \geq 0, \lambda_{1} \geq 0, \lambda_{2} \geq 0$ are fixed parameters, $c_{1}$ and $c_{2}$ are the average intensity values of pixels inside and outside contour $\mathcal{C}$ respectively. This approach is capable of dealing with low contrast objects, blurry edges or noisy image that cause failure in many gradient based segmentation methods. Furthermore, choosing a large value for $\mu$ in the above formulation encourages linking disconnected boundaries through conceptual edges. However, due to background noise and overlapping of different structures which commonly exist in medical images, the intensity values of vascular structures and the background are varying from regions to regions. Minimizing the energy functional can lead to a situation that the bright regions of background and dark portions of vessels belong to the same object.

Although approaches in [5] and [6] are robust to intensity variation of objects and background, they are confused by the fluctuating gradient of object boundaries in such case. The locally defined flux cannot recover the weak edges that are longer than the radius of the target object. Similarly, the elastic model is insensitive to small gradient of weak edges. This can lead to contour leakage. Besides, noise also generates intensity gradient across thin objects. It creates small gaps (discontinuities) on those narrow structures. The approaches above do not encode with the information about contour continuity. They tend to regard those single objects with small gaps as separated and disconnected structures.

In this paper, we propose a new vector field to incorporate with the active contour models for image segmentation. Calculation of the vector field is based on satisfying minimal weighted local variance calculated from the statistics after applying a set of filters on the image. Under this formulation, the magnitude and direction of an edge are not depending on its local gradient but the statistics estimated from a local region. The advantage of our method is that edges are extended along their direction so that the discontinued portion of the edges can be recovered without blurring or shifting effect. It is essential to recover those weak parts of edges in order to prevent contour leakage and discontinuity.

\section{Methodology}

\subsection{The Proposed Model}

Let $g(x, y)$ be a spatial filter which has its peak value at the center and decays gradually away from the center, for instance, Gaussian filter. We split the filter $g(x, y)$ into two filter sets according to a parameter $\theta, \theta \in[0, \pi)$. Each filter should be summed to one. Namely, $g_{1}(x, y, \theta)$ and $g_{2}(x, y, \theta)$ are defined as,

$$
g_{1}(x, y, \theta)=\frac{g_{1}^{\prime}(x, y, \theta)}{\int g_{1}^{\prime}\left(x^{\prime}, y^{\prime}, \theta\right) d x^{\prime} d y^{\prime}}
$$




$$
\begin{aligned}
g_{2}(x, y, \theta) & =\frac{g_{2}^{\prime}(x, y, \theta)}{\int g_{2}^{\prime}\left(x^{\prime}, y^{\prime}, \theta\right) d x^{\prime} d y^{\prime}}, \\
g_{1}^{\prime}(x, y, \theta) & =\left\{\begin{array}{cl}
g_{\theta}(x, y) & \text { if }(x, y)^{T} \cdot \hat{n_{\theta}}<0, \\
0 & \text { otherwise, }
\end{array}\right. \\
g_{2}^{\prime}(x, y, \theta) & =\left\{\begin{array}{cl}
g_{\theta}(x, y) & \text { if }(x, y)^{T} \cdot \hat{n_{\theta}}>0, \\
0 & \text { otherwise, }
\end{array}\right.
\end{aligned}
$$

where $\hat{n_{\theta}}=(\cos \theta, \sin \theta)^{T}$, and $g_{\theta}(x, y)=g\left(x \cos \left(\theta+\frac{\pi}{2}\right)-y \sin \left(\theta+\frac{\pi}{2}\right), x \sin (\theta+\right.$ $\left.\left.\frac{\pi}{2}\right)+y \cos \left(\theta+\frac{\pi}{2}\right)\right)$ is the rotated version of $g(x, y)($ Fig 13$)$.

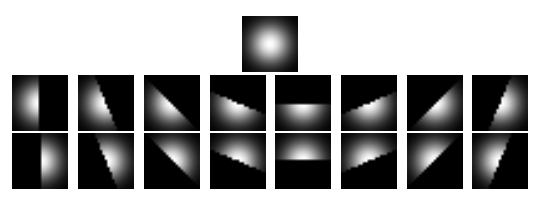

(a)

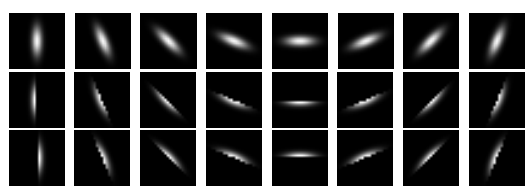

(b)

Fig. 1. (a) Top: $G_{\sigma=4}$. Second row: Corresponding filter set $g_{1}$. Third row: Corresponding filter set $g_{2}$. From left to right, $\theta=0, \frac{\pi}{8}, \frac{2 \pi}{8}, \frac{3 \pi}{8}, \frac{4 \pi}{8}, \frac{5 \pi}{8}, \frac{6 \pi}{8}, \frac{7 \pi}{8}$. (b) First row: $G_{\sigma_{x}=3, \sigma_{y}=1}$. Second row: Corresponding filter set $g_{1}$. Third row: Corresponding filter set $g_{2}$. From left to right, $\theta=0, \frac{\pi}{8}, \frac{2 \pi}{8}, \frac{3 \pi}{8}, \frac{4 \pi}{8}, \frac{5 \pi}{8}, \frac{6 \pi}{8}, \frac{7 \pi}{8}$.

We obtain $\theta^{\prime}(x, y)$ satisfying the following condition,

$$
\begin{aligned}
\theta^{\prime}(x, y)= & \arg \min _{\theta}\left\{\int \left\{g_{1}\left(x^{\prime}-x, y^{\prime}-y, \theta\right) \cdot\left(I\left(x^{\prime}, y^{\prime}\right)-\mu_{1}(x, y, \theta)\right)^{2}\right.\right. \\
& \left.\left.+g_{2}\left(x^{\prime}-x, y^{\prime}-y, \theta\right) \cdot\left(I\left(x^{\prime}, y^{\prime}\right)-\mu_{2}(x, y, \theta)\right)^{2}\right\} d x^{\prime} d y^{\prime}\right\} .
\end{aligned}
$$

Here $\mu_{1}=\int g_{1}\left(x^{\prime}-x, y^{\prime}-y, \theta\right) I\left(x^{\prime}, y^{\prime}\right) d x^{\prime} d y^{\prime}$ and $\mu_{2}=\int g_{2}\left(x^{\prime}-x, y^{\prime}-\right.$ $y, \theta) I\left(x^{\prime}, y^{\prime}\right) d x^{\prime} d y^{\prime}$. The terms $\mu_{1}(x, y)$ and $\mu_{2}(x, y)$ are the weighted averages of the neighboring pixels of $(x, y)$ in different sides split by the line $\hat{n}_{\perp \theta(x, y)}=$ $\left(\cos \left(\theta(x, y)+\frac{\pi}{2}\right), \sin \left(\theta(x, y)+\frac{\pi}{2}\right)\right)^{T}$. Equation (5) is the weighted sum variance of the neighboring pixels from the both sides of the line $\hat{n}_{\perp \theta(x, y)}$. We call this condition, minimal weighted local variance.

Now we define the vector field, $\mathcal{V}$, using the $\theta^{\prime}(x, y)$ obtained from minimal weighted local variance, $\mathcal{V}(x, y)$ is found as follows,

$$
\mathcal{V}(x, y)=\left\{\mu_{2}\left(x, y, \theta^{\prime}(x, y)\right)-\mu_{1}\left(x, y, \theta^{\prime}(x, y)\right)\right\} \cdot \hat{n}_{\theta^{\prime}(x, y)}
$$

By finding $\theta^{\prime}(x, y)$ that satisfies the minimal weighted local variance condition, the direction of $\mathcal{V}(x, y)$ is pointing from one region to another region such that the weighted sum variance of these two regions is minimized. Its magnitude is determined by the difference of weighted averages of these two regions. 


\section{$2.2 \quad$ Properties}

The main goal of defining $\theta^{\prime}(x, y)$ is to find the direction pointed by the vector $\hat{n}_{\perp \theta^{\prime}(x, y)}$, that is the best choice to partition the neighborhood of the pixel $(x, y)$ into two regions. For an ideal sharp edge that separates two regions with distinct constant intensity, $\mathcal{V}(x, y)$ gives similar results to the smoothed intensity gradient vector of the edge.

The major difference of the above formulation and Chan's 7 minimal partition problem is that we localized the calculation of variance and the contour evolution is guided by the direction of $\hat{n}_{\perp \theta^{\prime}(x, y)}$ at every point. For medical images, intensity of an object such as blood vessel is largely varying from regions to regions. Therefore, the sum variance of objects and background is not necessary to be minimal for correct segmentation result in those situations. Since the intensity variance of objects themselves is also large, minimizing the sum variance of inside and outside contours causes oversensitivity on those objects whose intensity is varying. In contrast, calculation of sum variance in a localized manner avoids this problem.

The localized sum variance of every pixel does not depend on the topology of contours. Instead, it depends only on the neighborhood of pixel. Obviously, the calculation of localized sum variance should be more sensitive to those pixels nearby and less sensitive to those pixels far away. The neighborhood is defined by the filter $g(x, y)$. A filter which has its peak value at the center and decays gradually away from the center is a good choice of $g(x, y)$, for example, Gaussian function.

In Equation (6), $|\mathcal{V}(x, y)|$ is given by $\mu_{2}\left(x, y, \theta^{\prime}(x, y)\right)-\mu_{1}\left(x, y, \theta^{\prime}(x, y)\right)$, which is the difference of weighted intensity average of the regions separated by $\hat{n}_{\perp \theta^{\prime}(x, y)}$. Such difference reflects how well the direction $\hat{n}_{\perp \theta^{\prime}(x, y)}$ partitions the regions around $(x, y)$.

Considering a vascular structure with fluctuating intensity value (Fig, 2a, b), the intensity of some segments of blood vessel is very similar to the background. Distinguishing those regions from background is difficult without referring to the neighbors. That's the reason why leakage problem commonly exists in different active contour models.

Those confusing regions can be recovered by referring to the intensity of pixel neighbors. In our formulation, $|\mathcal{V}|$ depends on the weighted intensity average difference of pixel neighbors. The filters $g_{1}\left(\cdot, \theta^{\prime}(x, y)\right)$ and $g_{2}\left(\cdot, \theta^{\prime}(x, y)\right)$ in (4) are chosen to be split along direction of $\hat{n}_{\perp \theta^{\prime}(x, y)}$, which is the tangent direction of boundaries extracted from minimal weighted local variance condition. Thus, $|\mathcal{V}(x, y)|$ are referring to two regions that are separated by a straight line along the direction of $\hat{n}_{\perp \theta^{\prime}(x, y)}$. It plays an important role in extending edges along their direction and links the boundaries that have coherent orientation.

On the other hand, for those weak edges having no coherent orientation to their neighbors, their field magnitude is suppressed by smoothing effect of weighted average. Linking weak edges are only performed for those boundaries with coherent edge direction. Thus, noise is suppressed which has weak interaction with its neighbors. 
In addition, our formulation only recovers those low contrast boundaries with high contrast edges along analogous direction in its neighborhood. Without considerable prior knowledge on the shape of target regions, the information extracted in minimal weighted local variance is not going to reconstruct those structures that are significantly confused with the background.

For vascular segmentation, target regions consist of elongated and thin structures. To make the field sensitive to these structures, we suggest to use a Gaussian kernel with different standard deviation in x and y directions, $G_{\sigma_{x}, \sigma_{y}}(x, y)=$ $\frac{1}{2 \pi \sigma_{x} \sigma_{y}} \exp \left\{-\frac{x^{2}}{2 \sigma_{x}^{2}}-\frac{y^{2}}{2 \sigma_{y}^{2}}\right\}$. The value of $\sigma_{x}$ controls the strength to extend boundaries along their direction, and $\sigma_{y}$ controls the width scale of the objects to be detected which should be set roughly smaller than the width of target structure. Large ratio of $\frac{\sigma_{x}}{\sigma_{y}}$ (Fig 10) makes calculation of weighted average and weighted variance consider less pixels along the direction of $\hat{n}_{\theta^{\prime}(x, y)}$ than direction of edges, $\hat{n}_{\perp \theta^{\prime}(x, y)}$. As a result, the vector field $\mathcal{V}$ calculated by such filter favors elongated objects such as blood vessels.

\subsection{Implementation}

The crucial step to estimate $\mathcal{V}(x, y)$ is to find $\theta^{\prime}(x, y)$. It can be achieved by defining a set of discrete values, $\theta_{k}$, where $k \in\{0, \ldots, K-1\}$ and $\theta_{k}=\frac{k \pi}{K}$. The $\theta^{\prime}(x, y)$ is obtained in discrete fashion,

$$
\begin{aligned}
\theta^{\prime}(x, y)=\arg \min _{\theta_{k}} & \left\{\sum _ { x ^ { \prime } , y ^ { \prime } } \left\{g_{1}\left(x^{\prime}-x, y^{\prime}-y, \theta_{k}\right) \cdot\left(I\left(x^{\prime}, y^{\prime}\right)-\mu_{1}\left(x, y, \theta_{k}\right)\right)^{2}\right.\right. \\
& \left.\left.+g_{2}\left(x^{\prime}-x, y^{\prime}-y, \theta_{k}\right) \cdot\left(I\left(x^{\prime}, y^{\prime}\right)-\mu_{2}\left(x, y, \theta_{k}\right)\right)^{2}\right\}\right\} .
\end{aligned}
$$

In our experiments, we have used $K=36$ for $\theta_{k} \in[0, \pi)$. Therefore, there are totally 36 filters for both $g_{1}\left(x, y, \theta_{k}\right)$ and $g_{2}\left(x, y, \theta_{k}\right)$ to detect 72 distinct edge orientations.

The vector field $\mathcal{V}(x, y)$ is then calculated by Equation (6) . It is defined in the whole image domain and is not affected by the dynamics of moving contours. We utilize the elastic model proposed in [6] to model the interaction between boundaries detected by the minimal weighted local variance. This model is used because of its long range interaction ability and high sensitivity to both concave and convex regions. Resulting contour is the minimizer of energy associated with moving contours and a vector field, w, as stated in Equation (2) subject to the constraint in Equation (3). Here we approximate $\delta_{\mathcal{C}} \mathbf{t}$ with $\delta(z) \cdot\left(v_{2},-v_{1}, 0\right)^{T}$ where $\mathcal{V}=\left(v_{1}, v_{2}\right)^{T}$ and use zero level of level set surface to represent moving contours 4], which is evolving according to the following equation,

$$
\phi_{t}=F|\nabla \phi|
$$

where $F$ is the normal velocity that the curve evolves. 
We can solve $F$ in frequency domain similar to [6]. $m, n, l$ denote the frequencies in $x, y$ and $z$ directions respectively and $\widetilde{F}(m, n, l)$ is the frequency component of $F(x, y) \cdot \delta(z)$,

$$
\widetilde{F}(m, n, l)=i \frac{m \cdot a_{1}(m, n)+n \cdot a_{2}(m, n)}{m^{2}+n^{2}+l^{2}},
$$

where $a_{1}(m, n)$ and $a_{2}(m, n)$ are frequency components of $v_{1}(x, y)$ and $v_{2}(x, y)$, respectively. Assume that the $3 \mathrm{D}$ space is continuous and extending to infinity in $z$ direction, discrete and periodic in both $x$ and $y$ directions,

$$
\begin{aligned}
F(x, y) & =\frac{1}{2 \pi} \int_{l=-\infty}^{l=\infty}\left\{\sum_{m, n} \frac{i \cdot\left(m a_{1}(m, n)+n a_{2}(m, n)\right) \cdot e^{i m x+i n y}}{m^{2}+n^{2}+l^{2}}\right\} d l, \\
& =\sum_{m, n} \frac{i\left(m a_{1}(m, n)+n a_{2}(m, n)\right)}{2 \sqrt{m^{2}+n^{2}}} e^{i m x+i n y} .
\end{aligned}
$$

Note that we have added a very small constant into the variable $m$ and $n$ in our implementation, which avoids singularity of the solution when $m$ and $n$ are both zero. The above formulation find $\left(v_{1 x}+v_{2 y}\right)$ and diffuses it to whole image domain with inverse square decay rate. The opposite sign of $\left(v_{1 x}+v_{2 y}\right)$ on two different sides over an edge creates zero-crossing boundary that halts the evolution of contour.

In [6], intensity gradient vector is used instead of $\mathcal{V}(x, y)$ in Equation (9). In this case, $a_{1}$ and $a_{2}$ are replaced with the frequency components of $-I_{x}$ and $I_{y}$ respectively. Finding the corresponding $\widetilde{F}$ is equivalent to applying the Laplacian filter on the image and diffusing it with inverse square decay rate. As a result, [6] is similar to the work in [8] about edge integration by finding zero-crossing after applying Laplacian filter on an image. It also has a close relationship with [5], where the Equation (11) is equivalent to $\mathcal{C}_{t}=\left(\nabla^{2} \cdot I\right) \mathcal{N}$.

Neither the inverse square decay rate of [6] nor discrete summation of Equation (11) over circular disc proposed in [5] carries information about contour continuity. In contrast, the minimal weighted local variance added those information by extending edges along their direction which is useful for segmentation of narrow and low contrast structures in noisy images.

Finally, to speed up evolution process, we replace $F(x, y)$ in Equation (8) with a sigmoid function $\frac{2}{1-e^{-F(x, y) / \sigma_{F}}}-1$ in our implementation, where $\sigma_{F}$ is the standard deviation of $F(x, y)$. This function has similar effect of $\operatorname{sign}(F)$ when magnitude of $F$ is very large while keeping increasing linearly as magnitude of $F$ is small.

\section{Experimental Results}

This section presents results obtained from real images (Fig 2) consisting of two digital subtraction angiography (DSA) obtained from the Department of Diagnostic Radiology and Organ Imaging, Prince of Wales Hospital, Hong Kong, 


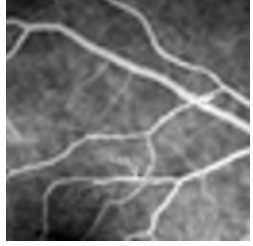

(a)

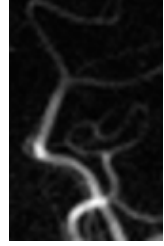

(b)

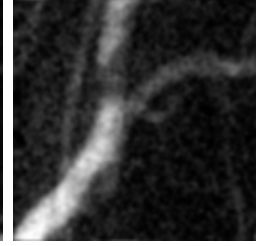

(c)

Fig. 2. (a) $128 \times 128$ retinal angiography. The intensity variation of vessel and background causes the gradient of vessel boundaries varying in different regions (top and bottom portion of the image). (b) $80 \times 128 \mathrm{DSA}$. Intensity of the object is dropped at the Y-shape and circular structure at the middle of the image. (c) $128 \times 128$ DSA. A portion of the vessel has relatively lower intensity than the other parts.

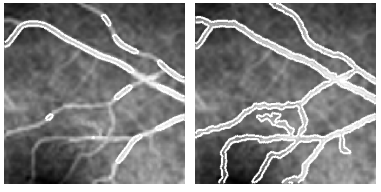

(a)

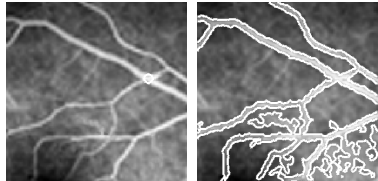

(b)
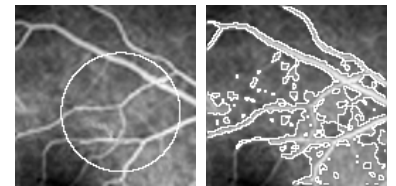

(c)

Fig. 3. Left: Initial contours. Right: Final results. (a): FM with $r=1,2,3$, image is preprocessed with $G_{\sigma=0.8}$, initial contour obtained automatically from regions with the highest $5 \%$ inward flux which is further smoothed under curvature flows for 200 steps. (b): ACM-EI, the image is preprocessed with $G_{\sigma=0.8}$, manually selected initial contour. (c): ACWE with $\mu=0.00001 \cdot 255^{2}, \lambda_{1}=\lambda_{2}=1, \nu=0, h=1$, manually selected initial contour.

and one retinal angiography [9]. Comparison is performed between the proposed method with three different approaches including "Flux Maximizing Geometric Flows" (FM) 5], "A New Active Contour Method based on Elastic Interaction" (ACM-EI) [6] and "Active Contours without Edges" (ACWE) [7.

The first example (Fig 2a) shows a retinal angiography. The background intensity is generally lower in left-bottom, left-top and right-top regions. Since the ACWE method partitions the image into high intensity group and low intensity
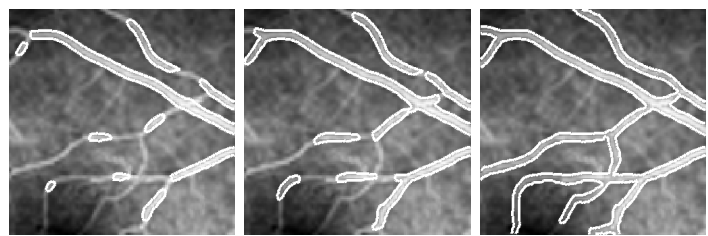

Fig. 4. Result of the proposed method using $\sigma_{x}=1.6$ and $\sigma_{y}=0.8$. Left: Initial contour obtained automatically from regions with the highest $5 \%$ field value which is further smoothed under curvature flows for 200 steps. Middle: Intermediate step. Right: Final result. 


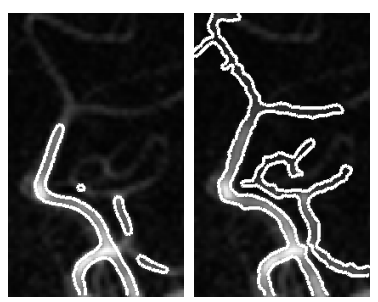

(a)

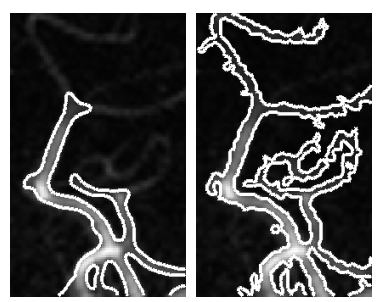

(b)
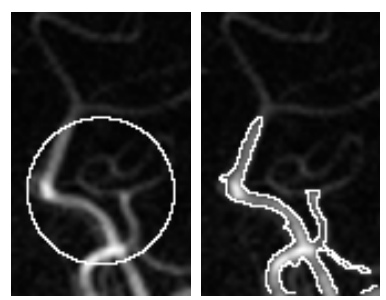

(c)

Fig. 5. Left: Initial contours. Right: Final results. (a): FM with $r=1,2,3$, image preprocessed with $G_{0.8}$, initial contour obtained automatically from regions with the highest $10 \%$ inward flux which is further smoothed under curvature flows for 200 steps. (b): ACM-EI initial contour obtained automatically using the heuristic approach presented in [6] with $\sigma_{1}=0.8$ and $\sigma_{2}=10$. (c): ACWE with $\mu=0.00001 \cdot 255^{2}, \lambda_{1}=$ $\lambda_{2}=1, \nu=0, h=1$, manually selected initial contour.

group, it cannot give a satisfactory result as the low intensity vessel is excluded from the contour while high intensity background is included (Fig 3 r).

On the other hand, ACM-EI tends to ignore weak edges when strong edges are present. Therefore, the contour is guided by noise and leaks through blurred boundaries at the bottom of the image (Fig $3 \mathrm{~b}$ ). We have manually placed the initial contour of ACM-EI inside the blood vessel as the heuristic approach in 6] cannot locate the vessel position in this low contrast situation. FM selects initial contour correctly and indicates side vessel as well (Fig 3 a). In contrast, our method favors smooth contour and keeps branches be connected without leakage. Fig 4 shows that our method locates the main vessel correctly, and can handle intensity variation in the object and background regions because of the calculation of minimal variance in a localized manner. It also avoids leakages in the low contrast regions since edges are extended along its direction.

In Fig,2b, we have shown a DSA where the intensity of the object is dropped significantly at two positions (the Y-shape structure and the circular structure at the middle of the image). As shown in Fig 5 , similar to the results previously shown, ACWE cannot capture objects in the dim regions. Besides, as shown
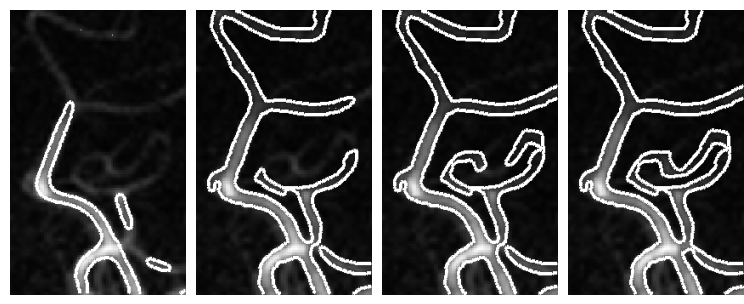

Fig. 6. Result of the proposed method using $\sigma_{x}=1.6$ and $\sigma_{y}=0.8$. Left: Initial contour obtained automatically from regions with the highest $10 \%$ field value which is further smoothed under curvature flows for 200 steps. Middle: Two intermediate steps, the contour is propagating through the narrow and dim segments. Right: Final result. 


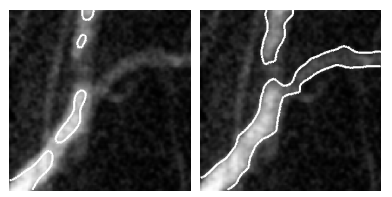

(a)

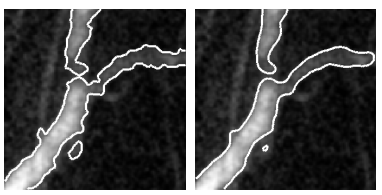

(b)
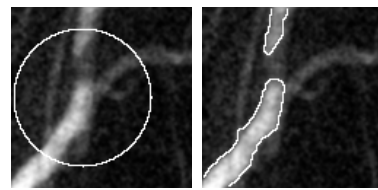

(c)

Fig. 7. Left: Initial contours. Right: Final results. (a): FM with $r=4,5,6,7$, image preprocessed with $G_{\sigma=3}$, initial contour obtained automatically from regions with the highest $5 \%$ inward flux which is further smoothed under curvature flows for 200 steps. (b): ACM-EI, initial contour obtained automatically using the heuristic approach presented in 6] with $\sigma_{1}=3, \sigma_{2}=10$. (c): ACWE with $\mu=0.4 \cdot 255^{2}, \lambda_{1}=\lambda_{2}=1, \nu=0, h=1$, manually selected initial contour.

in Fig $[5$, the contour of FM is halted when the gradient along the vessel is comparable to the gradient of object boundaries.

ACM-EI can capture the vessel but the result is noisy (Fig [5]), although it is the best results obtained among different combinations of parameters. The contour follows the noisy regions attached to the vessels rather than the weak vessel boundaries. Increasing either the $\sigma$ of the Gaussian filter or curvature term as authors suggested in [6] dose not help and results in contour halted at dim or narrow parts. In contrast, our method extends boundaries along their direction to recover the discontinued boundaries over dim and tiny segments. Thus, the contour can propagate through the dim and narrow regions (Fig 6).

The last example (Fig 2 ) shows a vessel with a dim portion. It aims to examine the ability of different approaches to connect a gap, which has size comparable to the object width. FM, ACM-EI and ACWE cannot merge the contours across the portion with low intensity value (Figs $7 \mathrm{a}$, b and c). The value of $\sigma$ of the Gaussian filter being used in ACM-EI and FM cannot be too large. Otherwise, they cannot handle the narrow branch at the right portion
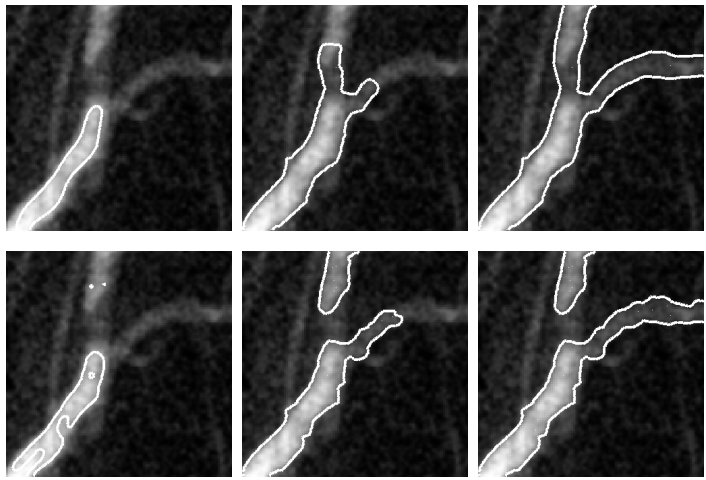

Fig. 8. Results of the proposed method using $\sigma_{x}=\sigma_{y}=3$ in the top row and $\sigma_{x}=$ $1, \sigma_{y}=3$ in the bottom row. Left: Initial contour obtained automatically from regions with the highest $5 \%$ field value which is further smoothed under curvature flows for 200 steps. Middle: Intermediate step. Right: Final result. 
of the image. ACWE fails to detect the narrow branch using different values of $\mu$ because of the significant intensity variation. Therefore, we only show the result with a large value of $\mu$, in which contour is not halted far away from vessel boundaries, as shown in Fig 7 f. It shows that ACWE cannot recognize the vessel as a single object. Here the proposed method is able to connect the top and bottom portions of the vessel (Fig $8 \mathrm{a}$ ). As mentioned in Section 2.2, a small value of $\sigma_{x}$ can be used to reduce the strength of boundary extension. We have demonstrated to use a small value of $\sigma_{x}$ for identifying the target region as separated objects in (Fig, $8 \mathrm{~b})$.

\section{Conclusion}

This paper proposed a new vector field for the detection of objects with narrow and elongated structures. The field is incorporated in the active contour models. The direction of boundaries is estimated based on the minimal weighted local variance condition, which extrapolates edges along their direction so that disconnected boundaries can be linked. In the experiments, the proposed method has been validated and compared to three different approaches. It is shown that the proposed method can effectively prevent contour leakage or discontinuity, which may happen in the segmentation of narrow structures with low contrast boundaries. Finally, our method is robust to intensity variation inside objects and background regions.

Acknowledgment. The authors would like to thank Dr. Yu of the Department of Diagnostic Radiology and Organ Imaging, Prince of Wales Hospital, Hong Kong, for providing the DSA images.

\section{References}

1. C. Xu and J. Prince, "Snakes, shapes, and gradient vector flow," IEEE T. Image Processing, (7):359-369, 1998.

2. M. Kass, A. Witkin, and D. Terzopoulos, "Snakes: Active contour models," Intern. J. Computer Vision, (1):321-331, 1988.

3. R. Malladi, J. Sethian, B. Vemuri, "Shape modeling with front propgagtion: A level set approach," IEEE T. PAMI., (17):158-175, 1995.

4. S. Osher, J. Sethian, "Fronts propagating with curvature dependent speed: algorithms based on hamilton-jacobi formulations," J. Comp. Phys., (79):12-49, 1988

5. A. Vasilevskiy, K. Siddiqi, "Flux Maximizing Geometric Flows" IEEE T. PAMI., (24):1565-1578, 2002.

6. Y. Xiang, A.C.S. Chung, J. Ye, "A New Active Contour Method based on Elastic Interaction," IEEE Conf. CVPR., (1):452-457, 2005.

7. T.F. Chan, L.A. Vese, "Active Contours without Edges," IEEE T. Image Processing, (10):266-277, 2001.

8. D. Marr, E. Hildreth, "Theory of Edge Detection," Proc. Royal Soc. of London, (B207):187-217, 1980.

9. Bonnie M. Gauer, OD, MS, "Using Fluorescein Angiography To Assess Retinal Disease," http://www.opt.pacificu.edu/ce/catalog/12059-PS/FA.html 